\title{
A decision making support tool: the Resilience Management Fuzzy Controller
}

\author{
J. Rubén G. Cárdenas ${ }^{1}$, Àngela Nebot ${ }^{2}$, Francisco Mugica², Alfredo Vellido ${ }^{2}$ \\ ${ }^{1}$ IUSS UME School Via Ferrata 45, Pavia, Italy \\ ${ }^{2}$ Soft Computing Group, Technical University of Catalonia, Jordi Girona Salgado 1-3, Barcelona, Spain \\ ruben.gonzalez@umeschool.it,angela@lsi.upc.edu,fmugica@lsi.upc.edu,avellido@cs.upc.edu
}

Keywords: Fuzzy Sets, Risk Management, Natural Hazards, Social Vulnerability, Resilience Management, Fuzzy Controllers, Inference System, Machine Learning, Artificial Intelligence, Decision Support System

\begin{abstract}
In this paper a fuzzy controller capable to perform an automated estimation of the period of time necessary to recover a resilience level is proposed. Estimations where made by considering realistic time-dependent action changes for a set of resilience indicators originally proposed by Cardona (2001) and modified by Cardenas et al (2015).The fuzzy resilience controller works using two output control variables and four input variables designed to resemble politics decisions made over resilience recovery while considering an economical national growth factor. We applied the fuzzy controller onto Barcelona Spain, where different recovery times where estimated in terms of variations in Spaniard GDP (Gross domestic product) inter anual rate of change. This Decision Support System might be helpful to assist disaster reduction planning by allowing decision takers, governs or institutions to achieve reliable recovery time estimations while a proper supervision and control of resilience indicators progress is performed and an open evaluation and scrutiny of applied policies is made.
\end{abstract}

\section{INTRODUCTION}

The concept of resilience has been broadly extended in order to reflect certain properties regarding the response capacity of a system altered either by an external or internal agent. However, due in part to the multiple disciplines on which such concept is applied and the diverse range of attributes that are used to assess it, there is no common agreement on a definition of resilience, which is followed by a disagreement over its metric and also, over the appropriate steps to achieve it.

A good example is disaster resilience, which is characterized through the response capacity of a social system to natural hazards but determined by the intrinsic relationship shaping every social entity, whether political, economic or environmental. There are several researches aimed to achieve resilience estimations, however only few studies have addressed the temporal dimension of disaster resilience, represented by the time lapse on which social sectors or subsystems return to a "normal" condition after a disturbance via recovery processes, as said by Brown et al., (2015). Such recovery times, inherently linked to a decision-making process, may be seen as a performance metric of the resilience capacity of the social system under scope (Gall, 2013). A proper assessment of recovery times is then a way to gain insights on how such decision framework can improve resilience in terms of an efficient management (Cimellaro et al., 2010).

As mentioned, resilience levels can be also deteriorated by subtler and not foreseen influences that will negatively alter response capacities. Such is the case of a narrowed economic landscape, which is capable to affect important aspects of resilience preparedness like efficiency and quality. This is especially true over those response bodies where an official budget is implemented. Clearly, these negative influences will be also reflected all over recovery process.

The aim of this study is to estimate the period necessary to restore the resilience level of a city after a fall on GDP values, considering factors representing institutional strengths entitled to deal with a seismic hazard, such as: the emergency operability level or the number of physicians. We included in the estimation components that might be catalogued as exclusively socials, such as development level. In order to achieve a more realistic estimation and be congruent with our assertions, all components are subject to different constrains, reflected either by economics restrains or by political issues. 


\section{THE FUZZY CONTROLLER}

The resilience fuzzy controller is in fact, a composition of two different controllers as showed in Figure 1; one controlling the changes to be done over values of the resilience indicator called human health resources (HHR) and the other one to control changes over resilience indicator called emergency operability (EO). The resilience controller uses fuzzy logic rules that relate input variables to those changes that would need to be made over output variables in order to achieve a particular resilience level. Once these changes have been established, they are used as inputs over a resilience fuzzy inference model (reported by Gonzalez et al., 2015) in order to achieve a final resilience estimation. A sample of these rules can be seen at the Appendix of this paper (Table 8). The resilience inference model is a classical fuzzy inference system type Mamdani whose performance is based in the development of fuzzy logic rules (if-then type) over the resilience indicators reported by Carreño et al., (2012); a sample of these rules can be seen at the Appendix of this paper (Table 7). The structure of the inference model resemble part of the theoretical holistic risk framework proposed by Cardona (2001), which considers that risk is a function of a physical component that is aggravated by social conditions, and the lack of resilience of the context. The set of resilience indicator are defined in Table 1, while a conceptualization of the inference model can be seen at Figure 2.

The fuzzy resilience controller initially works with four input variables: the difference between the actual resilience level and the resilience target $\left[R_{T}-R_{R}\right]$ (to be set by the user), the resilience tendency $\left[R_{R}-\right.$ $R_{R-1}$ ], relative to the previous unit of time (in this study: months), the Gross Domestic Product (GDP) increment and the Priority. These last controller input variables were defined in order to simulate two different types of constrains: an economical state indicator (GDP) which may be limiting resources allocation onto resilience improvement parameters, and a political constrain called "Priority", representing political willingness to improve resilience. With the use of such concepts we are attempting to simulate a real recovery planning scenario, where both types of constrains are always present.

Output control variables reflects the change in human health resources (HHR) and emergency operability (EO) that would need to be made relative to the previous month in order to drive resilience level to its desired final goal. Once the control is activated, final resilience level will totally depend on the control and the initial HHR and EO conditions will no longer followed. The domains of the four input variables are represented with fuzzy sets, that allow handling such concepts linguistically. All used membership function where Gaussian shaped. The range of actions of the input and output controller variables where selected considering real situations. For example, GDP increment range was defined considering real Spaniard GDP yearly percentage increments reported in literature, while $\triangle H H R$ was defined as the average change rate of physicians (at yearly base, per 1000 inhabitants) over the last 10 years in Barcelona city. In the case of EO, we are considering the number of police officers and fire-fighters change in the same interval. This information was obtained from the registered physicians evolution in Barcelona, as reported in the Barcelona Medic Council [http://www.comb.cat/cast/ciutadans/directori.htm] and from the Statistical Department of Barcelona Council respectively. The action range of the controller input variables that are related to the resilience, i.e. resilience distance to target and resilience tendency, where defined to be in the closed interval $[0,1]$ and $[-1,1]$, respectively. Table 2 and 3 shows input and output controller variables, their corresponding domains and actions ranges.

\section{RECOVERY TIMES ESTIMATION}

We designed two different experiments. The first one simulates consequences over Barcelona's city resilience level because of a significant fall of Spain's GDP during a limited period of time. By using the Fuzzy Inference System called "Resilience" described in section 2, those indicators used as inputs in the resilience FIS would react to this economic stress by decreasing their particular values as the maximum permitted by their reported average change rates, within a time interval arbitrarily selected. The second experiment was designed to achieve a particular resilience level through the simulation of control actions over two of the resilience indicators assumed as inputs in FIS Resilience. These control actions were in turn restricted by reported indicators change rates, budget constraints and official (political) commitment.

Both experiments are related. The estimated final values of Human Health Resources (HHR), Emergency Operability (EO), and Resilience level after a free fall on GDP's values (first experiment), will be used as the initial conditions to simulate the time necessary to readjust resilience level once a GDP increase has taken place (second experiment). The structure of the complete exercise is showed in Figure 3. 


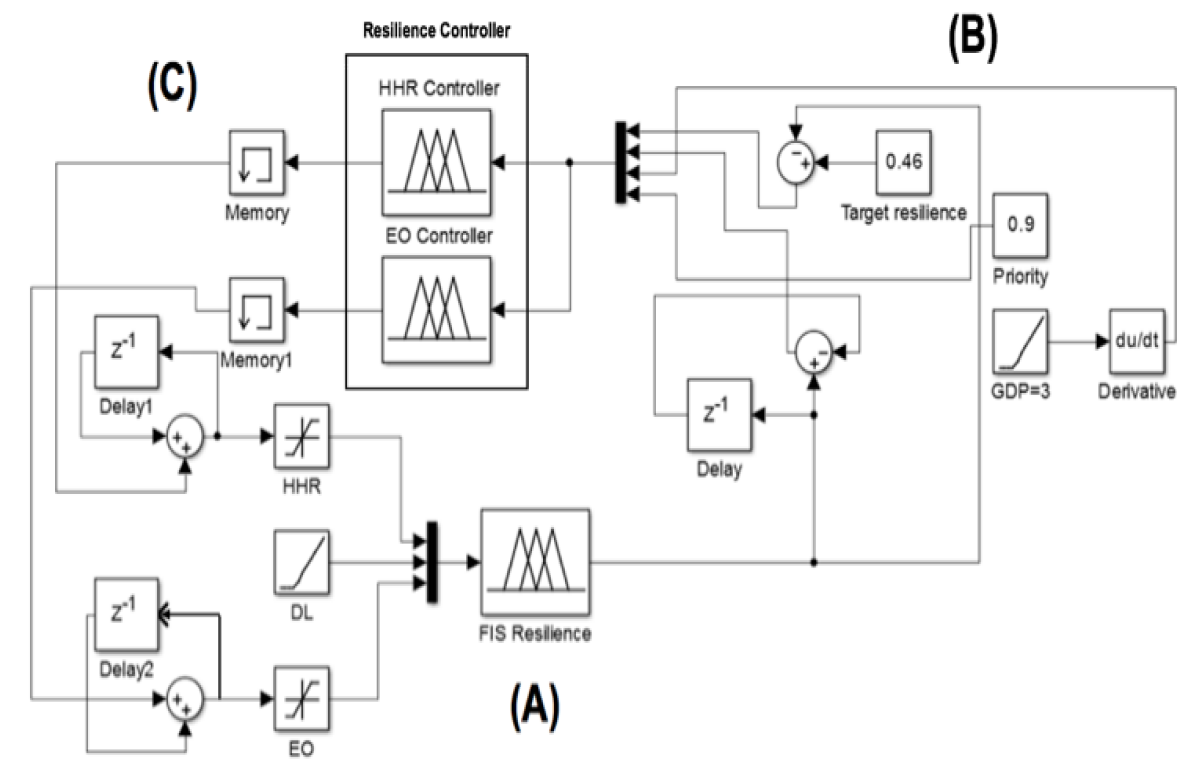

Figure 1: Conceptualization of the resilience fuzzy controller closed-loop operation. (A) A resilience level is estimated, (B) Input control variables are selected, in this example: $\mathrm{GDP}=3$ (medium), Priority $=0.9$ (high) and resilience target of 0.46 . (C) Control actions are taken.

Table 1: Input resilience indicators used in the fuzzy inference system (FIS) called Resilience (Cardenas et al., 2015) and adapted from Carreño et al., (2014)

Indicators

Human Health Resources (HHR)

Defined as the number of medical personnel per 1000 inhabitants. A greater amount of qualified personnel would mean a better way to manage a crisis situation.

Emergency Operability (EO)

Defined as the level of the capacity and preparation for emergency responding

in terms of the number of police personnel (in Spanish Guardia Urbana) and firefighters per 1000 inhabitants.

Development Level (DL)

Defined as the life quality level, planning and urban organization in the area.

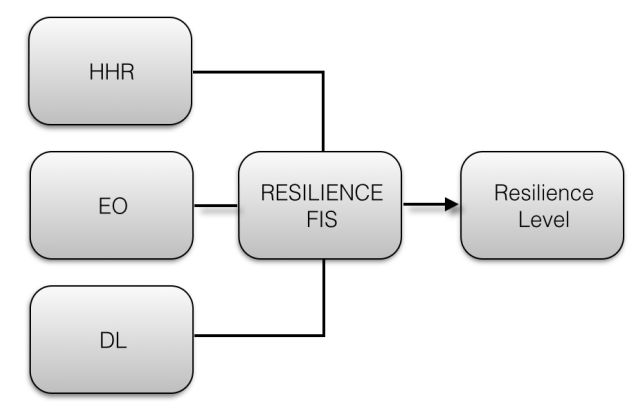

Figure 2: Structure of the fuzzy inference systems called Resilience. HHR=Human Health Resources, EO= Emergency Operability, DL= Development Level

\subsection{Resilience Simulation}

In order to model a maintained fall in GDP over time we selected a simple GDP's dynamics described by the expression $y=m$, where $m$ is equal to GDP change rate over a period of time and $y$ is the resulting GDP value. We defined two decreasing rates which where based in the more intense reported Spain's GDP decrease (inter annual variation) during the economical recession period of 2008-2010 which was $m_{1}=-0.4 \%$. The second rate was then defined as being half of the previous one, i.e. $m_{2}=-0.2$. In the same way, we are assuming that resilience's indicator changes in consequence following a constant rate as expressed by equation 1 and 2 .

$$
\begin{gathered}
\frac{d(H H R)}{d t}=\frac{d(G D P)}{d t}+\Delta H H R \\
\frac{d(E O)}{d t}=\frac{d(G D P)}{d t}+\Delta E O
\end{gathered}
$$

We selected an arbitrary time interval of 150 
Table 2: Inputs variables used in the fuzzy resilience controller. The table shows as well the action range, and the defined domain representation for each variable. Here $R_{T}$ stands for Resilience Target and $R_{R}$ for Resilience Real

\begin{tabular}{llc}
\hline Input control variables & Action Range & Fuzzy Sets \\
\hline$R_{T}-R_{R}$ & {$[0,1]$} & [stable, close, far] \\
$R_{R}-R_{R-1}$ & {$[-1,1]$} & [low, medium, high] \\
GDP increment & {$[0 \%, 8 \%]$} & [decreasing, stable, increasing] \\
Priority & {$[0,1]$} & [low, medium, high] \\
\hline
\end{tabular}

Table 3: Output variables used in the fuzzy resilience controller. The table shows as well the action range, and the defined domain representation for each variable

\begin{tabular}{ccr}
\hline Control Output Variables & Action Range & Fuzzy Sets \\
\hline$\Delta H H R$ & {$[0-0,08]$} & $\begin{array}{r}\text { [Stable, low-increment, } \\
\text { low-medium increment, }\end{array}$ \\
& & medium-high increment, high increment] \\
\hline$\Delta E O$ & {$[0-0,008]$} & [Stable, low-increment, \\
low-medium increment, \\
\end{tabular}

months or 12.4 years since we consider that this temporal window is wider enough to appreciate significant changes in resilience evolution. The initial values of HHR and EO where obtained by considering linguistic classes of medium for each indicator, as defined in the original resilience FIS. A summary of the parameters used in experiment 1 can be seen in Table 4.

Table 4: Parameters used on Experiment 1 using a time interval of 12.5 years for GDP change

\begin{tabular}{cc}
\hline GDP Parameters & Resilience Paramenters \\
\hline Change rate & HHR decreasing rate \\
i) $m_{1}=-0.2$ & $\Delta=0.083$ \\
ii) $m_{2}=-0.4$ & Initial value $=13$ \\
Init. value $=240$ bill. $\$$ & EO decre. rate \\
Time Interval $=$ & $\Delta=0.0083$ \\
150 months & Initial value=1 \\
& Resilience initerval \\
& value $=0.429$ \\
\hline
\end{tabular}

According to these linear considerations over GDP change rate, resilience indicators will change differently. For example: if $G D P_{m}=-0.2$; HHR and EO will reach values of 10.7 physicians/1000 inhabitants and $0.77 \mathrm{EO} / 1000$ inhabitants in 12.5 years of continuous fall of GDP nominal value, which would represent a diminish of $-23 \%$ of emergency bodies presence and $-18 \%$ for physicians respecting the initial conditions. In the case of $G D P_{m}=-0.4$, HHR will reach $-30 \%$ while EO will fall in $-52 \%$ respectively. These final estimations can be seen in Table 5. Figure 4 shows resilience final values in these scenarios. In the case of $G D P_{m}=-0.2$, the final resilience value will fall up to 0.354 , that represent a fall of $-31 \%$ whit respect of the initial resilience value. In the case of $G D P_{m}=-0.4$, resilience decrease will be of $-37 \%$.

\subsection{Fuzzy Control}

In order to simulate a control scheme over the resilience level, we simulated different scenarios through varying linguistic values of GDP and Priority input variables and selecting a particular resilience target level. By combining these elements, a customized control over resilience level can be established, for example: with a high GDP increase and a low Priority, would be feasible to achieve a resilience target of class medium in a time lapse of 200 months (or 16.7 years)? Evidently, the same question might be reformulated if now a high resilience level is desired, while the same input variables classes (linguistic values) remain unchanged or are modified.

We repeated these experiments by considering HHR and EO values obtained in experiment 1 , when GDP falls with a rate of $\mathrm{m}=-0.2$ or $\mathrm{m}=-0.4$, respectively. In this way we can estimate how different recovery times would be, when such recovery starts from conditions coming from a 'normal' economics recession, and when the recovery starts from an economic national (or even global) crisis situation. A constant value of 3 was given to the indicator named Development Level, corresponding to the linguistic class of medium as defined in the original Resilience Fuzzy Inference System. Table 6 shows just a small sample of the multiples scenarios (not included here due space reasons) that where used to execute control actions by assuming both economic conditions previ- 


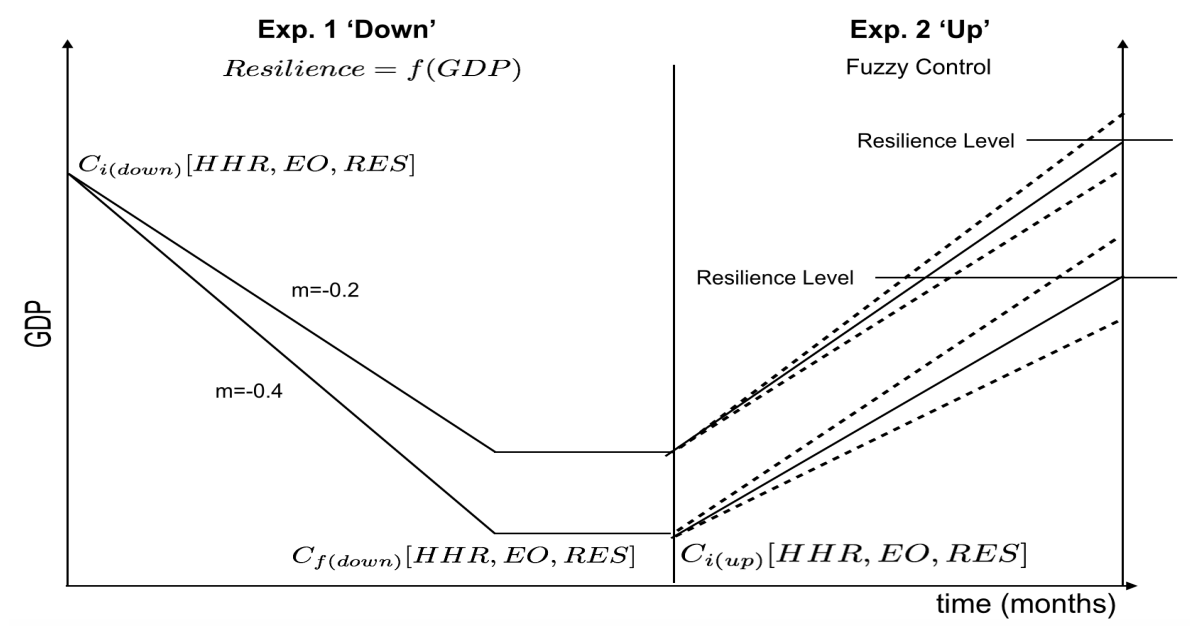

Figure 3: Structure of the experiments: (left) Experiment 1: Starting from initial condition of HHR, EO, and resilience level, $\left(C_{i(\text { down })}\right)$ along with Spain GDP value for 2015, two different slopes for GDP decreasing where used. Each of these will define two different sets of final resilience conditions $\left(C_{f}\right)$ that where used as initial condition for Experiment 2.

Table 5: Experiment 1, reached values for GDP, HHR, EO and resilience for a period of 150 months, where $m$ indicates GDP's slope

\begin{tabular}{lll}
\hline GDP reached values & Resilience reached values & \\
\hline Decreasing rate & HHR & HHR \\
i) $\mathrm{m}=-0.2$ & $G D P_{m}=-0.2$ & $G D P_{m}=-0.4$ \\
Initial value $=240$ bill. $\$$ & Initial value $=13$ & Initial value $=13$ \\
Final value $=210$ bill. $\$$ & Final value $=10.7$ & Final value $=8$ \\
\hline Decreasing rate & EO & EO \\
i) $m=-0.2$ & $G D P_{m}=-0.2$ & $G D P_{m}=-0.4$ \\
Initial value $=240$ bill. $\$$ & Initial value $=1$ & Initial value $=1$ \\
Final value $=180$ bill. $\$$ & Final value $=0.77$ & Final value $=0.451$ \\
& Resilience final value $=0.356$ & Resilience final value $=0.271$ \\
\hline
\end{tabular}

ously enunciated. Such flexibility gives to the fuzzy controller a suitable advantage in terms of the degree of freedom that a final user would have in order to select, design or modify its own required scenarios.

\section{Results}

Figure 5 shows estimated recovery times after a GDP fall of -0.2 and -0.4 respectively considering all depicted scenarios, but having as a resilience target level the original resilience level estimated before any GDP fall simulation (Table 4).

We can immediately note that as long as the economic resources and the official commitment remain high, the improvement of resilience level will be achieved in a shorter time. At the contrary, with poor official compromise and less economics resources, resilience recovery times increase substantially. From the previous figures we can note that there was only one estimation, under the 'worst' scenario, which reached a target resilience level within the considered time lapse.

According to our results, the economic well-being of a city is determinant to define a resilience recovery time. It is very different a city under economical recession than a city stressed by an economic crisis, where the resilience recovery time can be enlarged by a factor of almost 10. For example, under the actual economic crisis in Spain, and if city managers and authorities agree in both: resources and commitment; a city like Barcelona would need more than 8 years to return to the estimated prior crisis resilience level, and 14 years to reach an 'ideal' resilience level (graph not showed in the paper). Of course, such recovery times may be reduced if the rates of physicians and emergencies bodies production increases (which is a critical part on resilience planning). In the case of a city that was not impacted by such strong economi- 

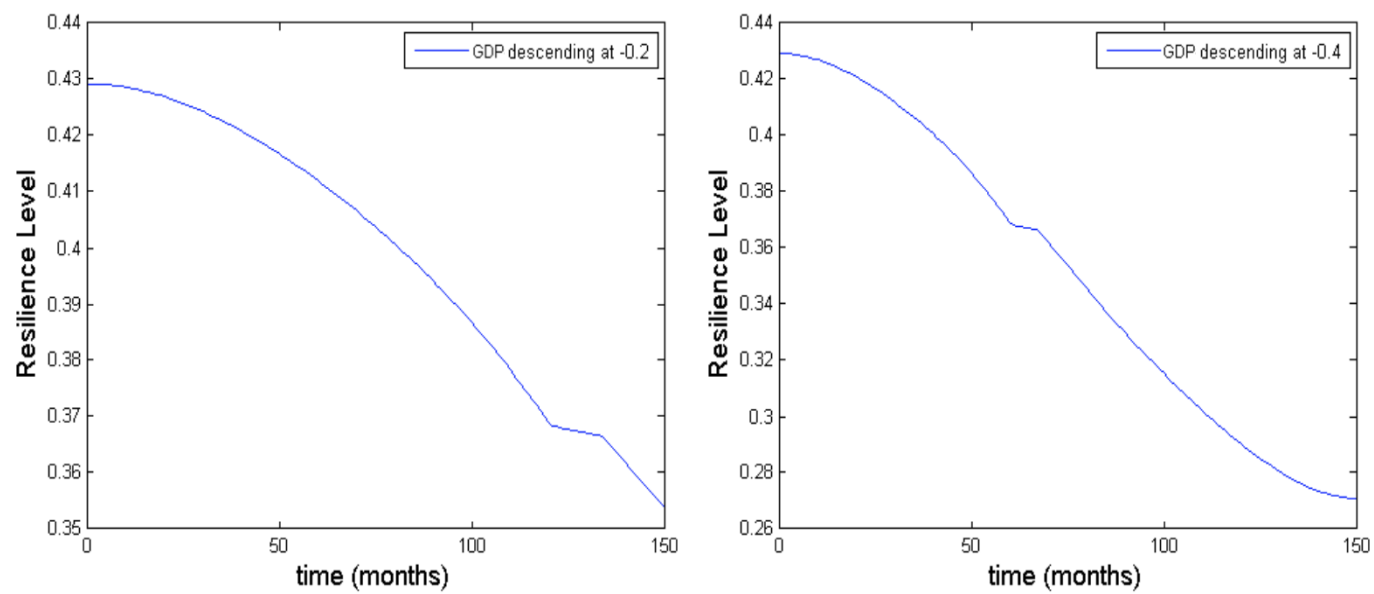

Figure 4: Resilience value reached if GDP goes down at $\mathrm{m}=-0.2$ (left), and $\mathrm{m}=-0.4$ (right)

Table 6: Experiments designed to estimate recovering times after a GDP fall of -0.2 : $\mathrm{DL}=3, \mathrm{EO}=0.75, \mathrm{HHR}=10.7$ while initial resilience value is 0.48 . In the case of a GDP fall of $-0.4 ; \mathrm{DL}=3, \mathrm{EO}=0.5, \mathrm{HHR}=8$ while the initial resilience value is 0.43 . Considered time laps was settled as $t=200$

\begin{tabular}{ll}
\hline $\begin{array}{l}\text { Scenario input } \\
\text { parameters }\end{array}$ & $\begin{array}{l}\text { Resilience to be } \\
\text { achieved }\end{array}$ \\
\hline $\begin{array}{l}\text { Worst Scenario } \\
\text { GDP } \equiv \text { low } \\
\text { Priority } \equiv \text { low }\end{array}$ & $\begin{array}{l}\text { Resilience Target } \\
\text { Original }\end{array}$ \\
\hline $\begin{array}{l}\text { Medium Scenario } \\
\text { GDP } \equiv \text { medium }\end{array}$ & $\begin{array}{l}\text { Resilience Target: } \\
\text { Original }\end{array}$ \\
Priority $\equiv$ medium & \\
\hline $\begin{array}{l}\text { Ideal Scenario } \\
\text { GDP } \equiv \text { high }\end{array}$ & Resilience Target: \\
Priority $\equiv$ high & Original \\
\hline
\end{tabular}

cal negative influences, recovery times will change in consequence.

\subsection{Using resilience controller}

It is crucial for resilience and risk management in general, to possess reliable estimations of recovery times while considering measurable factors. Using the resilience controller, recovery projections can be made in a long-medium term, while considering how an economic growth will translate into a better recovery performance, which is shaped by the institutional willingness to apply economic resources onto resilience improvement. Moreover, since the main controller's engine are merely the action range for the output control variables (that is: human health resources and emergency operability), a direct budget allocation can be planned in order to widen such ranges, influencing in consequence recovery time. In this way, the resilience fuzzy controller can be used to identify and exactly measure which are those critical action range increments that can be realistically achieved within a time interval, if a certain resilience level wants to be achieved.

Taking advantage of fuzzy methodologies, the resilience fuzzy controller can be easily spatiallyescalated either upwards or downwards, thus providing reliable recovery times estimations wether at national, municipality or at housing level. Our current work relies on the development of a Graphical User Interface (GUI) in order to provide a practical tool helpful to assist decision takers. For example, once the controller be properly adapted and improved, a final user would just need to select its target resilience level, include its resilience parameters (such as indicators, or resilience indices) and their production rates (considering also economic budget), and the resilience fuzzy controller will be capable to produce reliable estimations of recovery times that can be contrasted with the politics of the city under scope in order to see if they are reachable in a reasonable time, and considering the economic resources at hand. At last, since the resilience fuzzy controller is based in one single module of the fuzzy seismic risk model reported at Cardenas et al (2015), an immediate future work is the development of a full Risk fuzzy controller.

\section{CONCLUSIONS}

We developed a Decision Support System in the form of a fuzzy controller. The designed tool can estimate recovery times for resilience at city level con- 

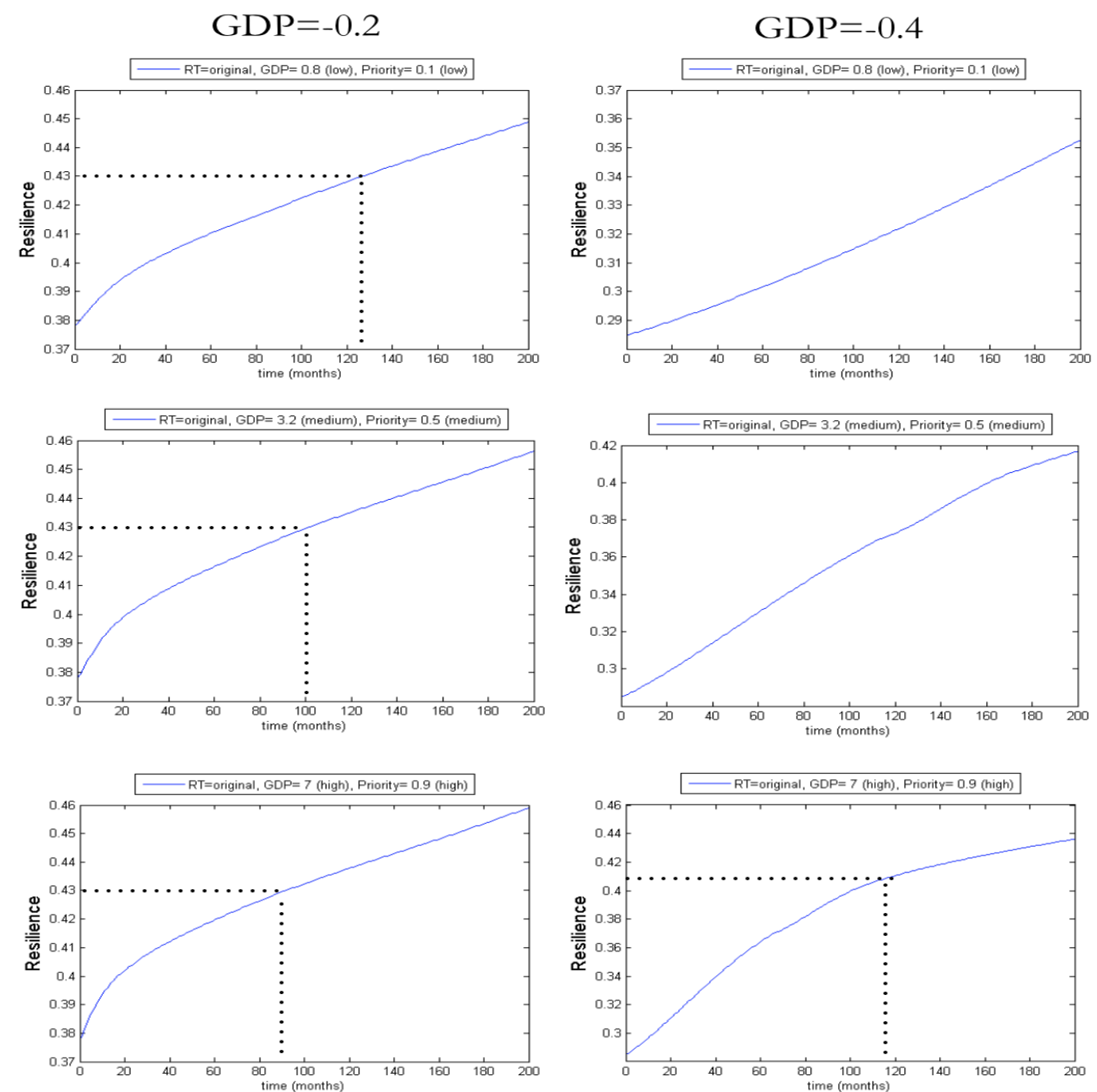

Figure 5: Estimated recovery times considering scenarios called: "worst", "medium", "ideal" and a resilience target level labeled as "original". Left column shows the amount of time required to return to the original resilience level, after a GDP fall of -0.2. Right column shows the amount of time required to return to the original resilience level, after a GDP fall of -0.4 . Dotted line shows the exact time at which the target resilience level has been achieved.

sidering an coerced economic landscape and political constrains. In this paper we estimated recovery times for the city of Barcelona, Spain by simulating a continuous fall on Spaniard GDP whose final result resemble those GDP values reported during the global economic crisis of 2008. These values allowed us to estimate subsequents resilience levels for the city by means of a fuzzy inference model, which is based on Cardona's holistic risk model (2001). In this way we were able to estimate the amount of time required to return to "normal" resilience levels by means of a fuzzy resilience controller, comprised by two individual fuzzy controllers working simultaneously over the values of Carreo (2012) resilience indicators. In order to be as realistic as possible, the action ranges conceded to resilience indicators uses only reported data easily accesible through Barcelona's Council statistical web page.

A main conclusion of the work presented here is that the economical well-being of a city is determinant to define a resilience recovery time. In this way, it is very different a city under economical recession than a city stressed by an economic crisis. In the case of Barcelona, 8 years are required to return to normal resilience levels, but 14 years are needed if resilience level wants to be improved. At the other hand, resilience fuzzy controller's configuration options allows to make projections under diverse circumstances 
that might be very helpful to answer questions such as: if a certain resilience level is required, what are those achievable changes that needs to be done onto resilience indicators? Or: in how long these changes would be reflected in a real resilience improvement?

\section{APPENDIX}

Table 7: Small sample of rules composing the resilience FIS model, used to estimate resilience level. $\mathrm{HHR}=\mathrm{Hu}-$ man Health Resources; DL = Development Level=cte; EO = Emergency Operability; $\mathrm{R}=$ Resilience; $\mathrm{VH}=$ very-high; $\mathrm{H}=$ high $; \mathrm{MH}=$ medium-high $; \mathrm{ML}=$ medium-low $; \mathrm{L}=$ low

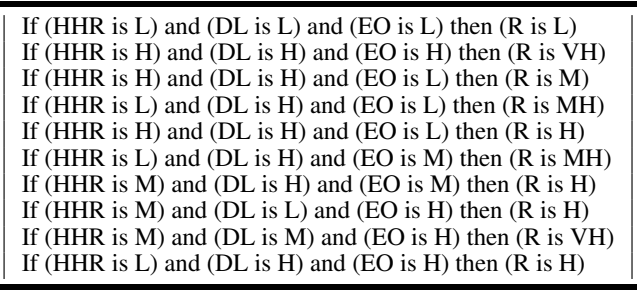

Table 8: Small sample of rules composing one of the two resilience fuzzy controller's components (human health resources, HHR). Rt-Rr stands for Resilience Target and $\mathrm{Rr}$ stands for Real or estimated Resilience

If (Rt-Rr is stable) and (Rr-Rr-1 is stable) and GDP

increment is (high) and Priority is (Low) then

(HHR is low-decrement).

If ( $\mathrm{Rt}-\mathrm{Rr}$ is close) and (Rr-Rr-1 is decreasing) and GDP

increment is (high) and Priority is (Low) then

(HHR is low-medium-increment).

If (Rt-Rr is far) and (Rr-Rr-1 is increasing) and GDP

increment is (low) and Priority is (Low) then (HHR is stable)

If (Rt- $\mathrm{Rr}$ is far) and (Rr-Rr-1 is decreasing) and GDP

increment is (low) and Priority is (Low) then

(HHR is low-increment)

If ( $\mathrm{Rt}-\mathrm{Rr}$ is close) and (Rr-Rr-1 is increasing) and GDP

increment is (high) and Priority is (medium) then

(HHR is low-medium-decrement)

If (Rt-Rr is stable) and (Rr-Rr-1 is decreasing) and GDP

increment is (medium) and Priority is (high) then

(HHR is low-increment).

If ( $\mathrm{Rt}-\mathrm{Rr}$ is close) and ( $\mathrm{Rr}-\mathrm{Rr}-1$ is decreasing) and GDP

increment is (low) and Priority is (medium) then

(HHR is medium-high-increment).

If ( $\mathrm{Rt}-\mathrm{Rr}$ is far) and (Rr-Rr-1 is stable) and GDP

increment is (medium) and Priority is (high) then

(HHR is high-increment).

If (Rt-Rr is far) and (Rr-Rr-1 is increasing) and GDP

increment is (low) and Priority is (medium) then

(HHR is low-medium-increment).

If (Rt-Rr is stable) and (Rr-Rr-1 is stable) and GDP

increment is (medium) and Priority is (medium) then

(HHR is medium-high-increment).

\section{References}

Brown E.D., William, B.K., (2015) Resilience and Resource Management. Environmental Management,56, 1416-1427.

Cutter, S.L., Boruff, B.J., Shirley, W.L., (2003) Social Vulnerability to Environmental Hazards. Social Science Quarterly,84 3639-3649.

Cimellaro, J.P., Reinhorn,M., Broneau, M., (2010) Framework for analytical quantification of disaster resilience. Engineering Structures,32 3639-3649.

Cardona, O.D., (2001), Holistic evaluation of the seismic risk using complex dynamic systems (in Spanish), $P h D$ Thesis Technical University of Catalonia, Barcelona, Spain. Cardona, O.D., (2003), The need for rethinking the concepts of Vulnerability and Risk from an Holistic Perspective: a necessary review a criticism for effective Risk Management, Mapping Vulnerability: Disasters, Developing and People Chapter 3 Earthscan Publishers, London.

Carreño, M.L. (2006), Innovating techniques for seismic risk evaluation and management in urban centers (in Spanish), PhD Thesis Technical University of Catalonia, Barcelona, Spain.

Carreño, M.L., Cardona, O.D., Barbat, A.H., (2007), Disaster risk management performance index, Nat Hazards 40 1-20.

Carreño, M.L., Cardona, O.D., Barbat, A.H., (2012) New methodology for urban seismic risk assessment from a holistic perspective Bull Earthquake Eng, 10, 547-565.

Gall, M., (2013) From Social Vulnerability to Resilience: Measuring Progress toward Disaster Risk Reduction United Nations University Institute for Environment and Human Security (UNU-EHS),ISBN: 978-3-944535-00-5.

Mamdani, E.H., Assilian, S., (1975) An experiment in linguistic synthesis with a fuzzy logic controller. Intern. J. of Man-Machine Studies, 7(1), 1-13.

Marulanda, M.C., Cardona, O.D., Barbat, A.H., (2009) Robustness of the holistic seismic risk evaluation in urban centers using the USRi, Nat Hazards, 49, 501-516.

Rose A., Krausmann, E., (2013) An economic framework for the development of a resilience index for business recovery International Journal of Disaster Risk Reduction, 5, 73-83. 